\title{
Das Ivanóczer Gebirg im südwestlichen Theile des Trencsiner Comitates.
}

\author{
Von Jos. Ludw. Holuby.
}

Schon seit längerer Zeit lockte mich das mannigfaltig geformte, von Podhragy nördlich gelegene Ivanóczer Gebirg, das meist aus grobkörnigem Sandstein bestehend schöne Buchenwälder tragt, and wenigstens e in en eintägigen Besuch, wenn nicht reichlich, doch zufriedenstellend zu lohnen versprach. Da ich aber his jetzt meine Aufmerksamkeit meist nur der Umgebung von N. Podhragy, Bosác, Haluzic, Stortek, Bohuslavicz, Mahrisch-Ljesko, Srnie und Mnesic, dem Dachsteinkalk-Berge Turecko und dem Wagthale geschenkt habe, deren Pflanzen ich sammle und notire, dagegen aber noch nie in den Ivanóczer Bergen gewesen bin, nahm ich mir einen verlässlichen Führer, und brach am 15. Mai um 6 Uhr Früh auf, um so gut es eben bei ein em Besuche geht, die Frühlingsflora des pyramidenförmigen, steilen Ivanóczer Gebirges kennen zu lernen.

Auf den nördlich von Podhragy gelegenen Kalkhügeln wurde ausser Carex Schreberi, Crepis praemorsa, Siler trilobum, Cerastium brachypetalum, Fragaria collina, Staphyllea pinnata, Pulmonaria mollis, und einigen anderen fast überall vorkommenden Pflanzen, nichts von Bedeutung gesehen. Von da richtete ich meine Schritte gegen Südost, um nachzusehen, was jetzt in der Nähe des Haluzicer Sauerbrunnen vorkommen könnte. Wie ich da auf morastigen Plätzen umherwate, überraschte mich die niedliche Salix angustifolia Wulf. mit längst verblühten weiblichen Kätzchen. Viele Arten Carices, deren Früchte ich noch abwarten will, dann Valeriana dioica, Arabis hirsuta, im Weidengebüsch Paris quadrifolia und üppige Exemplare der Listera ovata war so ziemlich alles, was des Erwähnens werth ist. Weiter östlich gegen das Ivanóczer Thal zu beobachtete ich Orchis Morio sehr spärlich, auf schlammigen Stellen Orchis latifolia, von den Knollenspitzen kaum 3 Zoll hoch, mit dicht gefleckten Blättern. Unter niedrigem Gebüsch wächst hier überall Hacquetia Epipactis, Sanicula europaea, Ranunculus auricomus, Carex pilosa und montana, Arum maculatum, Isopyrum thalictroides, das mir auf allen meinen Exkursionen begegnete bis an die Spitze des Lopennik. Im Ivanóczer Thal, welches ich in nordwestlicher Richtung durchstrich, konnte ich mich nicht lange aufhalten, um zum Besteigen des steilen Ivanóczer Gebirges nicht zu ermüden, und notire nur, was mir in Vorbeigehen unter die Augen kam. Beim Ostrolucky'schen Meierhof stand am Bache hie und da ein Exemplar des Ranunculus lanuginosus. Auf Wiesen bemerkte ich Carex tomentosa, glauca, panicea, Salix cinerea, purpurea, Viburnum Opulus, Caltha palustris, Eriophorum angustifolium, Viola pratensis, und einige andere Kosmopoliten, so dass ich fast ans Umkehren gedacht hätle, wäre mir nicht

Oesterr, botan. Zeitschrift. 8. Heft. 1865. 
Cardamine amara und Convallaria multiflora, dann Ranunculus polyanthem. $\beta$. angustisectus $\mathrm{N}$ eilr, aufgefallen.

Hier verliess ich das Thal, und richtete meine Schrilte den entwaldeten Stellen des Ivanóczer Gebirges zu. Von beiden Seiten des da hinauf führenden Weges wächst Asperula odorata in Menge, Fragaria vesca und elatior; am Bache Chaerophyllum temulum, Galeobdolon luteum, Oxalis Acetosella, Paris quadrifolia, Dipsacus pilosus nach den trockenen Stengeln vom vorigen Jahre erkennbar. Bevor man zu der lichten Stelle komml, muss man eine Waldparthie durchwandern, wo alte, schöne, riesengrosse Buchen stehen, wie man sie in den nahegelegenen Bosácer Wäldern vergebens suchen würde. Die darunter wachsenden baumartigen Corylus Avellana, Sambucus nigra, Ulmus effusa, Salix capraea und Acer Pseudoplatanus, erscheinen als unbedeutende Zwerge im Vergleich zu den Riesenbuchen. An der lichten Stelle stehen üppige Atropa Belladonna, Taraxacum officinale und einige andere Pflanzen der Niederung, die hierher geschleppt wurden. Ganze Strecken sind mit Rubus Idaeus and wahrscheinlich R. hirtus bewachsen, darunter das noch nicht blühende Epilobium angustifolium. Wohl selten triffl es zu, dass man unsere drei SambucusArten, nämlich S. racemosa vorherrschend, S. nigra minder häufig, und S. Ebulus, so beisammen findet, wie diess hier der Fall ist. Nur Schade, dass ich S. racemosa nicht mehr blühend angetroffen habe, und nur verblühte Exemplare für mein Herbar mitnehmen musste, da jch sie aus dieser Gegend noch nicht besass. Von dem, das Thal bewässernden Bache bis zu dieser Stelle kann man den Uebergang der Glechoma hederaceum in Gl. hirsutum W. K. beobachten, und es erscheinen Formen, die man mit eben solchem Rechte zu der ersteren wie zur letzteren ziehen kann. Junge Pflänzchen der Impatiens nolitangere bilden an feuchten Stellen ganze Rasen. Veronicu officinalis, wie ich sie bis jetzt nirgends gesehen habe, wächst sehr üppig. an Stellen, wo einst Kohlen gebrannt wurden. Myosotis sylvatica, Cynoglossum montanum und verblühete Dentaria bulbifera, Rasen von Carex maxima, und einzelne Exemplare von Ranunculus polyanthem. $\beta$, angustisectus $\mathrm{N}$ eil $\mathbf{r}$. wäre so ziemlich Alles, was diese entwaldete Stelle bietet.

Am Rücken dieses Gebirges wächst in grosser Menge Dentaria bulbifera, blässer und dunkler gefärbt, dagegen fehlt hier gänzlich D. enneaphyllos, die am Lopennik so häufig vorkommt. Mercurialis perennis, die in der Blattform sehr veränderlich ist, dann Corydalis cava, viel seltener C, solida, Cephalantera ensifolia - die einzige Orchidee, welche ich vom Ivanóczer Thal bis zur Spitze des Ivanóczer Gebirges bemerkie - wachsen hier gesellschaftlich. Am Wege sah ich Cerastium triviale, das auch sonst wo häufig anzutreffen ist. Prunus avium, im Thale längst verblüht, steht hier in Blüthenfülle. Auch wurden einige alte Sträuche von Crataegus oxyacantha, seltener $C r$. monogyna beobachtet. Längs diesem Kamme, stieg ich, am Wege immer dieselben Pflanzen begegnend, bis zur mährischen Gränze, wo man durch die weite Aussicht nach Mähren, dann auf das nördli- 
che Trentschiner, das Thúroczer, Neutraer Gebirg, namentlich westlich auf die Javorina, erfreut wird. Hier notirte ich noch Salvia glutinosa, die sehr häufig vorkomml, aber jetzl noch nicht blüht, Galizum vernum, viel seltener als auf den niedrigen Podhragyer Kalkhügeln; Hacquetia Epipactis, an einem kleinen Grasplatze Astrantia major, Viola silvestris, Daphne Mezereum, Chrysosplenium alternifolium, Ajuga reptans, sogar einige Exemplare Arabis Thaliana.

$\mathrm{Da}$ die Zeit schon bedeutend vorgeschritten war, musste ich eilen den Rückweg anzutreten, umsomehr, da ich noch das tiefe enge Thal Predpolomou, das schon zu Bosác gehört, besuchen wollte. Kaum dass man das Ivanóczer Gebirg verlüsst und das Bosácer betritt, wird man auch gewahr, dass in dem letzteren sehr übel mit den Wäldern gewirthschaftet wird. Es wäre wünschenswerth, wenn die Grundherrschaft mit allem Nachdrucke darauf bestehen möchte, die Commassation der Wälder zu beschleunigen, und so dem gänzlichen Verderben derselben Einhalt zu thun. Denn jetzt, besonders in den Monaten December und Jänner, fällt ein jeder Bosácer Insasse und Grundherr so viel, so oft, wo und was er will, junge und alte Bäume: so dass, wenn diess nur einige Jahre noch fortdauern möchte, man schwerlich e in en brauchbaren Baum finden würde.

Kann ich auch nicht sagen, dass dieser Besuch sehr lohnend war, bin ich mit dem Wenigen zufrieden, und hoffe, sollte ich einmal im Sommer Zeit gewinnen, mehr sammeln zu können.

Wie man das Thal Predpolomou betritt, ändert sich auch in einigem die Vegetation, und bietet mehr Mannigfaltigkeit. Von Farrn sah ich im Tvanóczer Gebirg nur das allerhäufigste Asp. Filix mas, dagegen hier in einer Schlucht, soviel ich jetzt unterscheiden vermochte: Asp. Filix mas, Cystopteris fragilis, Polypodium Dryopteris und Phegopteris. Der kommende Sommer wird zeigen, was da sonst noch an Filices zu finden sei. Die Unterlage daselbst ist schon Wiener Sandstein, und wenn man den Bach verfolgen will, muss man vorsichtig von einer Platte auf die andere steigen, um nicht etwa ein unfreiwilliges Bad im Bache zu nehmen. Aber mein Umherklettern war doch belohnt, denn gerade hier an schatligen Stellen fand ich Cardamine silvatica und impatiens, noch einige Schritle weiter $C$. amara, die unter allen die häufigste ist und bis tiel herab zum Dorfe N. Podhragy steigt.

"Pred polomou" sah ich auf Wiesen die erste Orchis militaris, die dann weiter südlich in grösserer Anzahl erscheint; Petasites officinalis truppenweise wachsend wird hier, verblüht über zwei Fuss hoch, wurde aber von mir bis jetzt nur in diesem Thale beobachtet. Am Bache bemerkte ich unter der sehr gemeinen Salix cinerea einige Sträuche S. amygdalina mit kolbenförmig verdickten männlichen Kätzchen, welche am Zweige fest silzen und später wahrscheinlich missgestaltete Blattbüschel bilden werden, wie dies namentlich bei $S$. fragilis und alba nicht selten der Fall ist. Bei einer Scheune überraschte mich das, sicher hieher geschlepple Asperugo procumbens, welche Pflanze in und um Podhragy bis jetzt nicht beobachtet wurde, 
dagegen schon in dem unweilen Bohuslavic vor dem gräfl. Bräunner'schen Castell häufig ist. Noch einmal wendete ich mich den Bergen zu, um von hier immer auf Wiesen steigend nach Hause zr kommen. Auf schlechten dem Norden zugekehrten Wiesen sammelte ich Ornithogolum umbellatum $\alpha$. silvestre Neilr. nahm noch einige Myosotis intermedia und silvatica, Carex tomentosa, Cerastium glutinosum. Listera ovata und Arabis hirsuta mit, und kam nach einem melr als zwölfstündigem Marsche nach Hause. Ich überzeugte mich, dass das Jvanóczer Gebirg eine weit ärmere Flora hat als der Lopennik, und was auf dem ersteren vorkommt, fehlt auch dem letzteren nicht. Ausserdem sind am Lopennik mehrere quellenreiche Stellen und Grasplätze, welche einen Ausflug, den ich noch diese Tage zu machen beabsichtige, gewiss besser lohnen werden!

Ns. Podhragy, am 17. Mai 1865.

\title{
Die europäischen Panicum-Arten.
}

\author{
Von Viktor v. Janka.
}

1. Flores singuli paniculati. 2.

Flores singuli spicati. 5 .

2. Spiculae acuminatae; plantae annuae. 3.

Spiculae acutae v. obtusiusculae; plantae perennes. 4.

3. Panicula erecta; spiculae minutae: Panioum capillare L.

Panicula cernua; spiculae majusculae: $\quad P$. miliaceum L.

4. Folia firma marginibus convoluta; rhizoma repens stoloniferum:

P. repens $\mathrm{L}$.

Folia mollia plana; rhizoma fibrosum: P. compressum Bir.

5. Spiculae paucae globoso-confertae spicam valde interruptam formantes; gluma utraque ex apice aristala:

P. undulatifolium R. et Sch.

Spiculae in spicas unilaterales nunc simplices digitatas vel verticillatas nunc compositas alternas dispositae; gluma utraque vel mutica vel superior solum aristata. 6 .

6. Spicae simplices anguste lineares 2-plures digitatae vel verticillatae. 7 .

Spicae compositae alternae. 10.

7. Gluma superior flores aequans vel superans. 8 .

Gluma superior floribus brevior:

$P$. sanguinale L. ( $P$. gracile G us s. enum. inarimens?

8. Spiculae acutae; gluma superior flores aequans. 9 .

Spiculae acuminatae; gluma superior flores superans:

$P$. debile Desf.

9. Spicae graciles; Spiculae ellipticae; culmi decumbentes haud radicantes; annuum:

P. glabrum Gaud. 